\title{
Polarization singularities in isotropic random vector waves
}

\author{
BY M. V. BERRY AND M. R. DENNIS \\ H. H. Wills Physics Laboratory, University of Bristol, \\ Tyndall Avenue, Bristol BS8 1TL, UK
}

Received 24 May 2000; accepted 8 August 2000

Following Nye \& Hajnal, we explore the geometry of complex vector waves by regarding them as a field of polarization ellipses. Singularities of this field are the $\mathrm{C}$ lines and $\mathrm{L}$ lines, where the polarization is purely circular and purely linear, respectively. The singularities can be reinterpreted as loci of photon spin 1 (C lines) and 0 (L lines). For Gaussian random superpositions of plane waves equidistributed in direction but with an arbitrary frequency spectrum, we calculate the density (length per unit volume) of $\mathrm{C}$ and $\mathrm{L}$ lines.

Keywords: singularities; polarization; optics; Gaussian; waves; randomness

\section{Introduction}

At each level of wave physics, singularities arise, and they are dissolved in the passage to a deeper level of description (Berry 2000). In geometrical optics, the singularities are caustics, that is, envelopes of families of rays (Arnold 1986; Berry \& Upstill 1980; Nye 1999). In complex scalar waves, the divergences at caustics are smoothed and decorated by diffraction. But the additional property that wave physics introduces (phase) has its own singularities, namely, dislocations or optical vortices (Nye \& Berry 1974; Nye 1999; Freund 2000), in the form of lines where the intensity vanishes (threads of darkness). When scalar waves are themselves an approximate description of a vector theory, as with light, dislocations are in turn dissolved. However, in the physics of vector waves, there is a further additional property (polarization) with its own line singularities, where the polarization is purely circular or purely linear (Nye 1991; Nye \& Hajnal 1987).

It is these singular lines in polarization fields that we will study here, with an emphasis on their statistics for vector waves that are random. Our aim is to generalize to vector singularities our recent calculations of statistics of dislocation lines (Berry \& Dennis 2000). This work is prompted by renewed interest in wave singularities, arising from interference experiments with lasers (Soskin 1997; Vasnetsov \& Staliunas 1999), and also by random wave fields that occur naturally, for example, black-body radiation.

Section 2 is largely a review of the work of Nye \& Hajnal (1987); we establish notation for complex vector waves, describe the polarization ellipse that relates the complex vectors to the real vectors and obtain conditions for the line singularities of polarization. In addition, we derive formulae for the directions of the lines. In $\S 3$ we reinterpret the polarization singularities in terms of photon spin operators and 
their eigenstates, and relate the local wavenumber to the local quantum expectation value of momentum. Section 4 introduces our statistical model of isotropic Gaussian random vector waves and the tools for calculating averages. The calculations of the averages themselves are carried out in $\S 5$; for the two sorts of polarization line singularity, we determine the average line length per unit volume (equal to twice the mean number of lines piercing unit area).

\section{Polarization ellipse and singularity geometry}

We consider time-dependent three-dimensional complex vector fields $\boldsymbol{E}(\boldsymbol{r}, t)$, which can be written in terms of their real and imaginary parts:

$$
\boldsymbol{E}(\boldsymbol{r}, t)=\boldsymbol{P}(\boldsymbol{r}, t)+\mathrm{i} \boldsymbol{Q}(\boldsymbol{r}, t) .
$$

(In the following, all $t$ dependence will be suppressed, and also all $\boldsymbol{r}$ dependence when this will not cause confusion.) $\boldsymbol{E}$ can, for example, be regarded as the complex electric field in a light wave, taken as the complex analytic signal (positive-frequency part) of the real field (Mandel \& Wolf 1995).

The polarization ellipse (Born \& Wolf 1959; Nye \& Hajnal 1987) is traced out by the vector

$$
\operatorname{Re} \boldsymbol{E} \exp (-\mathrm{i} \chi)=\boldsymbol{P} \cos \chi+\boldsymbol{Q} \sin \chi
$$

as the phase $\chi$ is varied. The vector sweeps out equal areas of the ellipse for equal intervals of $\chi$, and $\boldsymbol{P}$ and $\boldsymbol{Q}$ are conjugate radii (that is, each is parallel to the other's tangent). Nye \& Hajnal (1987) consider monochromatic waves, for which $\chi$ is proportional to time, so the ellipse is repeatedly traced out in real time. There is a special value $\chi=\chi_{0}(\bmod \pi)$, such that $\boldsymbol{E} \exp \left(-\mathrm{i} \chi_{0}\right)=\boldsymbol{P}_{0}+\mathrm{i} \boldsymbol{Q}_{0}$, where $\boldsymbol{P}_{0}$ and $\boldsymbol{Q}_{0}$ are the (orthogonal) major and minor semiaxes of the ellipse. We thus have a field of polarization ellipses (changing if the waves are non-monochromatic), with the ellipse at each point lying in the $\boldsymbol{P}, \boldsymbol{Q}$-plane.

The polarization ellipse at each point in space has an orientation given by the unit normal $\boldsymbol{n}_{\mathrm{e}}(\boldsymbol{r})$, which also defines the direction of increasing $\chi$ (that is, the sense of circulation of the ellipse). $\boldsymbol{n}_{\mathrm{e}}(\boldsymbol{r})$ is given by

$$
\boldsymbol{N}_{\mathrm{e}}(\boldsymbol{r}) \equiv N_{\mathrm{e}}(\boldsymbol{r}) \boldsymbol{n}_{\mathrm{e}}(\boldsymbol{r})=\frac{1}{2} \operatorname{Im}[\boldsymbol{E} *(\boldsymbol{r}) \times \boldsymbol{E}(\boldsymbol{r})]=\boldsymbol{P}(\boldsymbol{r}) \times \boldsymbol{Q}(\boldsymbol{r}) .
$$

Thus the ellipse field also defines a field of orthogonal frames $\left\{\boldsymbol{P}_{0}(\boldsymbol{r}), \boldsymbol{Q}_{0}(\boldsymbol{r}), \boldsymbol{n}_{\mathrm{e}}(\boldsymbol{r})\right\}$.

There are two types of line singularity of the polarization field. The first is where the polarization is purely circular. For the ellipse $(2.2)$ to be a circle, the real vectors $\boldsymbol{P}$ and $\boldsymbol{Q}$ must be equal in length and orthogonal. These are two conditions, so loci of pure circular polarization have codimension two and so are lines in space; we refer to them as C lines (Nye \& Hajnal (1987) used the term $\mathrm{C}^{\mathrm{T}}$ lines, with $\mathrm{T}$ denoting 'true', to avoid confusion with analogous singularities in paraxial fields (Nye 1983)). The singularity in this case is the orientation of the axes of the ellipse, which is not uniquely defined for a circle, so the phase $\chi_{0}$ cannot be defined on a C line.

With the definition of a complex scalar field

$$
\psi \equiv \boldsymbol{E} \cdot \boldsymbol{E} \equiv \xi+\mathrm{i} \eta=P^{2}-Q^{2}+2 \mathrm{i} \boldsymbol{P} \cdot \boldsymbol{Q},
$$


the $\mathrm{C}$ line condition implies

$$
\psi(\boldsymbol{r})=0 \quad(\boldsymbol{r} \text { on a } \mathrm{C} \text { line })
$$

(that is, $\boldsymbol{E}$ is nilpotent).

Since a $\mathrm{C}$ line is the intersection of the zero surfaces $\xi=0$ and $\eta=0$, the unit direction $\boldsymbol{n}_{\mathrm{C}}$ of the line, which lies in both surfaces, is perpendicular to their two normals, and so is given by

$$
\boldsymbol{N}_{\mathrm{C}} \equiv N_{\mathrm{C}} \boldsymbol{n}_{\mathrm{C}}=\nabla \xi \times \nabla \eta .
$$

A C line is generically a singularity with index $\pm \frac{1}{2}$, about which the local pattern of (nearly circular) ellipses rotates by half a turn (this is consistent with $\mathrm{C}$ being an index \pm 1 dislocation singularity of $\psi$, because $\psi$ involves $\boldsymbol{E}$ quadratically).

The second type of singularity is where the polarization is purely linear. For the ellipse (2.2) to be a line, the vectors $\boldsymbol{P}$ and $\boldsymbol{Q}$ must be (anti)parallel, so the complex vector $\boldsymbol{E}$ is the product of a real vector and a complex phase factor. We denote the unit direction of this real vector (parallel to $\boldsymbol{P}, \boldsymbol{Q}$ ) by $\boldsymbol{e}_{\boldsymbol{P}}$. Parallelism of vectors corresponds to two conditions, so loci of pure linear polarization have codimension two and thus are lines in space; we shall refer to them as L lines (Nye \& Hajnal used the term $\mathrm{L}^{\mathrm{T}}$ lines). The $\mathrm{L}$ condition implies that, in terms of (2.3),

$$
\boldsymbol{N}_{\mathrm{e}}(\boldsymbol{r})=\mathbf{0} \quad(\boldsymbol{r} \text { on an L line }) .
$$

This implies that an $\mathrm{L}$ line is a singularity with index \pm 1 in the plane perpendicular to $\boldsymbol{e}_{\boldsymbol{P}}$, about which the local pattern of vectors $\boldsymbol{N}_{\mathrm{e}}$ rotates by a full turn.

In Appendix A we show that the direction $\boldsymbol{n}_{\mathrm{L}}$ of the $\mathrm{L}$ line is given by

$$
\boldsymbol{N}_{\mathrm{L}} \equiv N_{\mathrm{L}} \boldsymbol{n}_{\mathrm{L}}=\frac{1}{2}\left(\nabla_{a} \times \nabla_{b}\right)\left(\boldsymbol{N}_{\mathrm{e}, a} \times \boldsymbol{N}_{\mathrm{e}, b} \cdot \boldsymbol{e}_{\boldsymbol{P}}\right),
$$

where the suffixes $a$ and $b$ indicate the vectors on which the $\nabla$ operators act.

$\mathrm{L}$ and $\mathrm{C}$ lines can be interpreted as degeneracies of the $\boldsymbol{r}$-dependent real symmetric matrix $P_{i} P_{j}+Q_{i} Q_{j}$, whose eigenvalues are $Q_{0}^{2}, P_{0}^{2}$ and zero, and whose eigenvectors constitute the frame $\boldsymbol{P}_{0}, \boldsymbol{Q}_{0}, \boldsymbol{n}_{\mathrm{e}}$. C lines are where the non-zero eigenvalues coincide and $\mathrm{L}$ lines are where the zero eigenvalue coincides with one of the others. Degeneracies of real symmetric matrices have codimension two (i.e. they are diabolical points (see, for example, Berry 1984, p. 50)), concordant with the fact that L and $\mathrm{C}$ singularities are lines.

\section{Relation with photon spin and momentum}

It is interesting (especially in view of recent re-examinations of photon angular momentum (Allen et al. 1999; Berry 1998; Simpson et al. 1997)) to recast the results of the preceding section in a manner that evokes the quantum description of light. This is based on the identity, for any vectors $\boldsymbol{A}$ and $\boldsymbol{B}$,

$$
\boldsymbol{A} \times \boldsymbol{B}=-\mathrm{i}(\boldsymbol{A} \cdot \hat{\boldsymbol{S}}) \boldsymbol{B}
$$

where the three components of the Hermitian vector operator $\hat{\boldsymbol{S}}$ can be represented by the following matrices, acting on the column vector $\boldsymbol{B}$ :

$$
\hat{\boldsymbol{S}}=\left\{\hat{S}_{x}, \hat{S}_{y}, \hat{S}_{z}\right\}=\left\{\left(\begin{array}{ccc}
0 & 0 & 0 \\
0 & 0 & \mathrm{i} \\
0 & -\mathrm{i} & 0
\end{array}\right),\left(\begin{array}{ccc}
0 & 0 & -\mathrm{i} \\
0 & 0 & 0 \\
\mathrm{i} & 0 & 0
\end{array}\right),\left(\begin{array}{ccc}
0 & \mathrm{i} & 0 \\
-\mathrm{i} & 0 & 0 \\
0 & 0 & 0
\end{array}\right)\right\}
$$


in terms of the antisymmetric symbol, $S_{i, j k}=\mathrm{i} \varepsilon_{i j k} . \hat{\boldsymbol{S}}$ satisfies the commutation relations for spin-1 quantum particles, namely (in units of $\hbar$ ),

$$
\hat{\boldsymbol{S}} \times \hat{\boldsymbol{S}}=\mathrm{i} \hat{\boldsymbol{S}}
$$

Now let the field $\boldsymbol{E}$ be regarded as a state, parametrized by $\boldsymbol{r}$ and $t$, and represented by its corresponding complex unit 3-vector $\boldsymbol{e}$, using the convenient Dirac notation

$$
\boldsymbol{e} \equiv \frac{\boldsymbol{E}}{\sqrt{\boldsymbol{E}^{*} \cdot \boldsymbol{E}}}, \quad\left(\begin{array}{c}
e_{x} \\
e_{y} \\
e_{z}
\end{array}\right) \equiv|\boldsymbol{e}\rangle, \quad\left(\begin{array}{ccc}
e_{x}^{*} & e_{y}^{*} & e_{z}^{*}
\end{array}\right) \equiv\langle\boldsymbol{e}|, \quad\left\langle\boldsymbol{e}^{\prime} \mid \boldsymbol{e}\right\rangle \equiv \boldsymbol{e}^{\prime *} \cdot \boldsymbol{e} .
$$

Then the expectation value $\boldsymbol{S}$ of the operator $\hat{\boldsymbol{S}}$ can be written (after using the antisymmetry of $\hat{\boldsymbol{S}}$ ) as

$$
\boldsymbol{S} \equiv\langle\boldsymbol{e}|\hat{\boldsymbol{S}}| \boldsymbol{e}\rangle=\operatorname{Im}\left(\boldsymbol{e}^{*} \times \boldsymbol{e}\right)=\frac{2 \boldsymbol{N}_{\mathrm{e}}}{\boldsymbol{E}^{*} \cdot \boldsymbol{E}}
$$

Thus the local spin state can be regarded as a vector normal to the polarization ellipse.

$\mathrm{C}$ and $\mathrm{L}$ lines now appear as loci of special spin values. We have (cf. equations (2.4) and (2.5))

$$
S^{2} \equiv \boldsymbol{S} \cdot \boldsymbol{S}=\left(-\mathrm{i} \boldsymbol{e}^{*} \times \boldsymbol{e}\right) \cdot\left(-\mathrm{i} \boldsymbol{e}^{*} \times \boldsymbol{e}\right)=1-|\boldsymbol{e} \cdot \boldsymbol{e}|^{2}=1 \text { on a C line. }
$$

Therefore, $\mathrm{C}$ lines correspond to spin-1 states; it is not hard to show that in this case, $|\boldsymbol{e}\rangle$ is an eigenstate of the operator $\boldsymbol{n}_{\mathrm{e}} \cdot \hat{\boldsymbol{S}}$, with eigenvalue +1 (the sign corresponds to the fact that $\boldsymbol{n}_{\mathrm{e}}$ gives the direction of circulation of $\boldsymbol{e}$ around the ellipse). For the L lines, equation (2.7) shows that the expectation $\boldsymbol{S}=0$, and indeed $|\boldsymbol{e}\rangle$ is an eigenstate of the operator $\boldsymbol{e} \cdot \hat{\boldsymbol{S}}$, with eigenvalue 0 . These results generalize the familiar relations between photon spin and the polarization of light fields.

It is worth remarking that the spin and Dirac notations enable the formula (2.8), for the direction of an $\mathrm{L}$ line, to be written in a slightly more transparent form, namely,

$$
\boldsymbol{N}_{\mathrm{L}}=\frac{1}{2} N_{\mathrm{e}}^{2} \operatorname{Im}\left\langle\nabla \boldsymbol{n}_{\mathrm{e}}\left|\times\left(\boldsymbol{e}_{\boldsymbol{P}} \cdot \hat{\boldsymbol{S}}\right)\right| \nabla \boldsymbol{n}_{\mathrm{e}}\right\rangle
$$

where the cross product connects the gradients.

Within the same framework, the natural definition of the wavevector $\boldsymbol{k}$ of the field $\boldsymbol{E}$ is as the local expectation of the momentum operator in the state $|\boldsymbol{e}\rangle$, namely,

$$
\boldsymbol{k} \equiv-\mathrm{i}\langle\boldsymbol{e}|\nabla| \boldsymbol{e}\rangle .
$$

As pointed out by Nye (1991), this is also the geometric phase 1-form connecting the fields at neighbouring points. Unlike the wavevector for a field of rays in geometrical optics, $\boldsymbol{k}$ is non-integrable. Indeed, there is a geometric phase $\gamma(\Gamma)$, defined by the integral of $\boldsymbol{k}$ round a circuit $\Gamma$ in $\boldsymbol{r}$, with the following meaning. Imagine a unit vector $\boldsymbol{e}^{\prime}$, equal to $\boldsymbol{e}$ at each point $\boldsymbol{r}$ apart from a phase that is determined by parallel transport from some starting point on $\Gamma$ where $\boldsymbol{e}^{\prime}=\boldsymbol{e}$, that is, by the rule $\left\langle\boldsymbol{e}^{\prime}|\nabla| \boldsymbol{e}\right\rangle=0$. Then $\gamma(\Gamma)$ is the phase difference between $\boldsymbol{e}^{\prime}$ and $\boldsymbol{e}$ at the end of the 
circuit. $\gamma(\Gamma)$ is also the flux through $\Gamma$ of a 2 -form, which can be written, using the suffix notation of (2.8), as

$$
\boldsymbol{D} \equiv \nabla \times \boldsymbol{k}=\operatorname{Im}\langle\nabla \boldsymbol{e}|\times| \nabla \boldsymbol{e}\rangle=\operatorname{Im} \nabla_{a} \times \nabla_{b} \boldsymbol{e}_{a}^{*} \cdot \boldsymbol{e}_{b}
$$

We have not found an interpretation of $\boldsymbol{D}$ in terms of the polarization geometry of the field $\boldsymbol{E}$. Singularities of $\boldsymbol{D}$, analogous to the codimension-3 monopoles of geometric phase theory (Berry 1984; Shapere \& Wilczek 1989), occur where the field strength $\boldsymbol{E}^{*} \cdot \boldsymbol{E}$ vanishes, but this is a codimension-6 phenomenon and so will not occur generically in a wave-field in space (or spacetime).

\section{Gaussian random vector waves}

Generalizing the scalar theory of Berry \& Dennis (2000), we write the complex vector wave as a superposition of many transverse plane waves with wavevectors $\boldsymbol{k}$, frequencies $\omega(\boldsymbol{k})$ and random phases $\mu_{\boldsymbol{k}}$,

$$
\boldsymbol{E}(\boldsymbol{r}, t)=\sum_{\boldsymbol{k}} a_{\boldsymbol{k}} \boldsymbol{n}_{\boldsymbol{k}} \exp \left\{\mathrm{i}\left(\boldsymbol{k} \cdot \boldsymbol{r}-\omega(\boldsymbol{k}) t+\mu_{\boldsymbol{k}}\right)\right\} .
$$

Each wave $\boldsymbol{k}$ is characterized by its real scalar amplitude $a_{\boldsymbol{k}}$ and the complex unit vector $\boldsymbol{n}_{\boldsymbol{k}}$, perpendicular to $\boldsymbol{k}$, representing its polarization. Thus $\boldsymbol{n}_{\boldsymbol{k}}^{*} \cdot \boldsymbol{n}_{\boldsymbol{k}}=1$, $\boldsymbol{k} \cdot \boldsymbol{n}_{\boldsymbol{k}}=0$ (so $\nabla \cdot \boldsymbol{E}=0$ ). Explicitly, using a temporary coordinate frame with axes 1,2 , with 3 along $\boldsymbol{k}$, and polar angles $\alpha$ and $\beta$ on the Poincaré polarization sphere (Born \& Wolf 1959):

$$
\boldsymbol{n}_{\boldsymbol{k}}=\left\{\cos \left(\frac{1}{2} \alpha_{\boldsymbol{k}}\right) \exp \left(-\frac{1}{2} \mathrm{i} \beta_{\boldsymbol{k}}\right), \sin \left(\frac{1}{2} \alpha_{\boldsymbol{k}}\right) \exp \left(\frac{1}{2} \mathrm{i} \beta_{\boldsymbol{k}}\right), 0\right\} .
$$

(We could also incorporate randomness in the amplitudes $a_{k}$, but this is not necessary.)

The distribution of $\boldsymbol{k}$ is assumed sufficiently dense for the sum (4.1) to represent an ensemble of Gaussian random functions, parametrized by $\mu_{\boldsymbol{k}}, \alpha_{\boldsymbol{k}}, \beta_{\boldsymbol{k}}$, with averaging defined by

$$
\langle\cdots\rangle \equiv \prod_{k} \underbrace{\frac{1}{2 \pi} \int_{0}^{2 \pi} \mathrm{d} \mu_{\boldsymbol{k}}}_{\text {phase average }} \underbrace{\frac{1}{4 \pi} \int_{0}^{\pi} \mathrm{d} \alpha_{\boldsymbol{k}} \sin \alpha_{\boldsymbol{k}} \int_{0}^{2 \pi} \mathrm{d} \beta_{\boldsymbol{k}}}_{\text {polarization average }} \cdots .
$$

(The assumption here, that polarizations are uniformly distributed on the Poincaré sphere for each plane-wave component, can be shown to be equivalent to independence of the Cartesian components of the complex $\left.\boldsymbol{n}_{\boldsymbol{k}}\right)$.

The power spectrum $\Pi(k)$ of the wave, for the case of isotropic randomness that we consider here, is defined by

$$
\sum_{\boldsymbol{k}} a_{\boldsymbol{k}}^{2} \cdots \equiv \int \mathrm{d} \boldsymbol{k} \frac{1}{4 \pi k^{2}} \Pi(k) \cdots,
$$

where here and hereafter $\mathrm{d} \boldsymbol{k}=\mathrm{d} k_{x} \mathrm{~d} k_{y} \mathrm{~d} k_{z}$, etc. The singularity densities to be calculated in $\S \S 5$ and 6 will depend on the second moment,

$$
k_{2} \equiv \int_{0}^{\infty} \mathrm{d} k k^{2} \Pi(k) / \int_{0}^{\infty} \mathrm{d} k \Pi(k) .
$$


Two important special cases are monochromatic radiation with wavenumber $k$, where obviously $k_{2}=k^{2}$, and black-body radiation with temperature $T$, for which (Berry \& Dennis 2000)

$$
k_{2}=\frac{40}{21} \pi^{2} k_{T}^{2} \equiv \frac{40}{21} \pi^{2}\left(\frac{k_{\mathrm{B}} T}{\hbar c}\right)^{2},
$$

where $k_{\mathrm{B}}$ is Boltzmann's constant.

To save writing, and without loss of generality, we use the normalization

$$
\int_{0}^{\infty} \mathrm{d} k \Pi(k)=1 .
$$

Thus

$$
\left\langle\boldsymbol{E}^{*} \cdot \boldsymbol{E}\right\rangle=1
$$

It is not hard to show from (4.2) and (4.3) that all components of the real and imaginary parts $\boldsymbol{P}$ and $\boldsymbol{Q}$ of $\boldsymbol{E}$ (cf. (2.1)) are independent and identically distributed random variables, with probability densities and variances

$$
\left.\begin{array}{c}
\rho(\boldsymbol{P})=\left(\frac{3}{\pi}\right)^{3 / 2} \exp \left(-3 P^{2}\right), \quad \rho(\boldsymbol{Q})=\left(\frac{3}{\pi}\right)^{3 / 2} \exp \left(-3 Q^{2}\right), \\
\left\langle P_{x}^{2}\right\rangle=\left\langle P_{y}^{2}\right\rangle=\cdots=\left\langle Q_{z}^{2}\right\rangle=\frac{1}{6} .
\end{array}\right\}
$$

We will also require the following averages over derivatives of $\boldsymbol{P}$ and $\boldsymbol{Q}$, derived in Appendix B:

$$
\left.\begin{array}{rl}
\left\langle\left(\partial_{x} P_{x}\right)^{2}\right\rangle & =\left\langle\left(\partial_{y} P_{y}\right)^{2}\right\rangle=\cdots=\frac{1}{30} k_{2} \\
\left\langle\left(\partial_{x} P_{y}\right)^{2}\right\rangle & =\left\langle\left(\partial_{y} P_{x}\right)^{2}\right\rangle=\cdots=\frac{1}{15} k_{2} \\
\left.\partial_{x} P_{x} \partial_{y} P_{y}\right\rangle & =\left\langle\partial_{x} P_{y} \partial_{y} P_{x}\right\rangle=\cdots=-\frac{1}{60} k_{2} .
\end{array}\right\}
$$

\section{Density of $\mathrm{C}$ lines}

From (2.5), C lines are defined by the vanishing of the complex scalar $\psi$ defined by (2.4) and hence by the vanishing of its real and imaginary parts $\xi$ and $\eta$. Integrating $\delta(\xi) \delta(\eta)$ over a volume, including the appropriate Jacobian, and averaging (see Berry \& Dennis (2000) for several examples of this procedure), we obtain, for the mean length of $\mathrm{C}$ line per unit volume,

$$
d_{\mathrm{C}}=\langle\delta(\xi) \delta(\eta)|\nabla \xi \times \nabla \eta|\rangle .
$$

The required averages involve the probability densities of $\boldsymbol{P}$ and $\boldsymbol{Q}$, and the gradients of these quantities. Although the $\delta$-functions are not independent of the Jacobian, the following stratagem enables the averages to be evaluated separately. We denote by $\rho(\boldsymbol{U}, \boldsymbol{V} ; \boldsymbol{P}, \boldsymbol{Q})$ the conditional probability density of $\boldsymbol{U} \equiv \nabla \xi$ and $\boldsymbol{V} \equiv \nabla \eta$ with $\boldsymbol{P}$ and $\boldsymbol{Q}$ fixed, that is, averaging only over the gradients of $\boldsymbol{P}$ and $\boldsymbol{Q}$, rather than the vectors themselves. Thus, in an obvious notation,

$$
\rho(\boldsymbol{U}, \boldsymbol{V} ; \boldsymbol{P}, \boldsymbol{Q}) \equiv\langle\delta(\boldsymbol{U}-\nabla \xi) \delta(\boldsymbol{V}-\nabla \eta)\rangle_{(\boldsymbol{P}, \boldsymbol{Q})} .
$$


Then (5.1) can be written

$$
\begin{aligned}
d_{\mathrm{C}}=\int \mathrm{d} \boldsymbol{P} \int \mathrm{d} \boldsymbol{Q} \rho(\boldsymbol{P}) \rho(\boldsymbol{Q}) \delta( & \left.P^{2}-Q^{2}\right) \delta(2 \boldsymbol{P} \cdot \boldsymbol{Q}) \\
& \times \int \mathrm{d} \boldsymbol{U} \int \mathrm{d} \boldsymbol{V} \rho(\boldsymbol{U}, \boldsymbol{V} ; \boldsymbol{P}, \boldsymbol{Q})|\boldsymbol{U} \times \boldsymbol{V}| .
\end{aligned}
$$

Writing the $\delta$-functions in (5.2) as Fourier integrals, and making use of the fact that the resulting exponent depends linearly on the Gaussian variables $\nabla P_{x}$, etc., we obtain

$$
\begin{aligned}
\rho(\boldsymbol{U}, \boldsymbol{V} ; \boldsymbol{P}, \boldsymbol{Q}) \equiv \frac{1}{(2 \pi)^{6}} \int \mathrm{d} \boldsymbol{s} \int \mathrm{d} \boldsymbol{t} \exp \{-\mathrm{i}(\boldsymbol{U} \cdot \boldsymbol{s}+\boldsymbol{V} \cdot \boldsymbol{t})\} \\
\times\langle\exp \{\mathrm{i}(\boldsymbol{s} \cdot \nabla \xi+\boldsymbol{t} \cdot \nabla \eta)\}\rangle_{(\boldsymbol{P}, \boldsymbol{Q})} \\
=\frac{1}{(2 \pi)^{6}} \int \mathrm{d} \boldsymbol{s} \int \mathrm{d} \boldsymbol{t} \exp \{-\mathrm{i}(\boldsymbol{U} \cdot \boldsymbol{s}+\boldsymbol{V} \cdot \boldsymbol{t})\} \exp \left\{-\frac{1}{2} T\right\},
\end{aligned}
$$

where (introducing an obvious suffix notation)

$$
T \equiv\left\langle(\boldsymbol{s} \cdot \nabla \xi+\boldsymbol{t} \cdot \nabla \eta)^{2}\right\rangle_{(\boldsymbol{P}, \boldsymbol{Q})}=4\left[\left(s_{i} s_{k}+t_{i} t_{k}\right)\left(P_{j} P_{l}+Q_{j} Q_{l}\right)\left\langle\partial_{i} P_{j} \partial_{k} P_{l}\right\rangle\right] .
$$

Anticipating the integrations over the $\delta$-functions in (5.3), we can incorporate the orthogonality and equal length of $\boldsymbol{P}$ and $\boldsymbol{Q}$, and exploit isotropy, by writing

$$
\boldsymbol{P}=(P, 0,0), \quad \boldsymbol{Q}=(0, P, 0) .
$$

Thus

$$
\begin{aligned}
T & =4 P^{2}\left[\left(s_{x}^{2}+s_{y}^{2}+t_{x}^{2}+t_{y}^{2}\right)\left(\left\langle\left(\partial_{x} P_{x}\right)^{2}\right\rangle+\left\langle\left(\partial_{x} P_{y}\right)^{2}\right\rangle\right)+2\left(s_{z}^{2}+t_{z}^{2}\right)\left\langle\left(\partial_{x} P_{y}\right)^{2}\right\rangle\right] \\
& =4 P^{2} k_{2}\left[\frac{1}{10}\left(s_{x}^{2}+s_{y}^{2}+t_{x}^{2}+t_{y}^{2}\right)+\frac{2}{15}\left(s_{z}^{2}+t_{z}^{2}\right)\right]
\end{aligned}
$$

where (4.10) has been used.

The $\boldsymbol{s}$ and $\boldsymbol{t}$ integrations in (5.4) are Gaussian, leading to

$$
\rho(\boldsymbol{U}, \boldsymbol{V} ; \boldsymbol{P}, \boldsymbol{Q})_{(P=Q, \boldsymbol{P} \cdot \boldsymbol{Q}=0)}=\frac{375}{256 \pi^{3} k_{2}^{3} P^{6}} \exp \left\{-\frac{5}{4 k_{2} P^{2}}\left(U^{2}+V^{2}-\frac{1}{4}\left(U_{z}^{2}+V_{z}^{2}\right)\right)\right\} .
$$

After rescaling $\boldsymbol{U}$ and $\boldsymbol{V}$, the $\boldsymbol{P}$ and $\boldsymbol{Q}$ integrations in (5.3) are elementary, and the $\mathrm{C}$ line density becomes

$$
d_{\mathrm{C}}=\frac{9 k_{2}}{320 \pi^{4}} \int \mathrm{d} \boldsymbol{U} \int \mathrm{d} \boldsymbol{V}|\boldsymbol{U} \times \boldsymbol{V}| \exp \left\{-\frac{1}{2}\left[U^{2}+V^{2}-\frac{1}{4}\left(U_{z}^{2}+U_{z}^{2}\right)\right]\right\} .
$$

In Appendix $\mathrm{C}$ this six-fold integral is evaluated, giving

$$
d_{\mathrm{C}}=k_{2}\left(\frac{3}{10 \pi}+\frac{1}{5 \sqrt{ } 3}\right)=0.21096 k_{2} .
$$

This is the main result of this section. We make three remarks.

(i) Just as for dislocation lines in scalar waves (Berry \& Dennis 2000), the mean density of points where $\mathrm{C}$ lines cross a plane is $\frac{1}{2} d_{\mathrm{C}}$. 
(ii) $\frac{1}{2} d_{\mathrm{C}}$ is close to the result $k_{2} /(3 \pi)=0.10610 k_{2}$ for the density (length per unit volume) of scalar dislocation lines (Berry \& Dennis 2000) (the appearance of $\frac{1}{2} d_{\mathrm{C}}$ is unrelated to that in (i)).

(iii) For monochromatic radiation with wavelength $\lambda=2 \pi / k$, and for black-body radiation with thermal wavelength $\lambda_{T}=2 \pi / k_{T}$ (cf. (4.6)), the densities are

$$
d_{\mathrm{C}}= \begin{cases}8.3283 / \lambda^{2}, & \text { monochromatic } \\ 156.57 / \lambda_{T}^{2}, & \text { black-body }\end{cases}
$$

\section{Density of $\mathrm{L}$ lines}

Following Appendix A, we consider an L line through $\boldsymbol{r}=\mathbf{0}$, for which $\boldsymbol{P}$ and $\boldsymbol{Q}$ have the common direction $\boldsymbol{e}_{\boldsymbol{P}}=\boldsymbol{e}_{z}$, and whose direction $\boldsymbol{n}_{\mathrm{L}}$ is parallel to $\boldsymbol{A} \times \boldsymbol{B}$, defined in (A 1) and (A 2). We seek the density which, when integrated over a small volume surrounding $\boldsymbol{r}=\mathbf{0}$, gives the length of $\mathrm{L}$ within the volume. From (A 2), $\delta(\boldsymbol{A} \cdot \boldsymbol{r})$ restricts the integral in $x$, and $\delta(\boldsymbol{B} \cdot \boldsymbol{r})$ restricts the integral in $y$, so the product $\delta(\boldsymbol{A} \cdot \boldsymbol{r}) \delta(\boldsymbol{B} \cdot \boldsymbol{r})$ restricts the integral to L; to give the correct density, it must be multiplied by the transverse Jacobian $\partial \boldsymbol{A} \cdot \boldsymbol{r} \partial \boldsymbol{B} \cdot \boldsymbol{r} / \partial x \partial y$, and divided by the cosine of the angle between $\boldsymbol{N}_{\mathrm{L}}$ and $\boldsymbol{e}_{\boldsymbol{z}}$. It is not hard to show that the required density is

$$
\delta(\boldsymbol{A} \cdot \boldsymbol{r}) \delta(\boldsymbol{B} \cdot \boldsymbol{r})|\boldsymbol{A} \times \boldsymbol{B}|
$$

(note that $|\boldsymbol{A} \times \boldsymbol{B}|$ is the length of the vector $\boldsymbol{N}_{\mathrm{L}}$ in (2.8)).

Now, $\boldsymbol{A} \cdot \boldsymbol{r}$ and $\boldsymbol{B} \cdot \boldsymbol{r}$ are the $x$ and $y$ components of the vector $\boldsymbol{P} \times \boldsymbol{Q}$ that lies in the $x y$-plane; therefore, we can use the identity

$$
\delta(a) \delta(b)=\frac{\delta\left(\sqrt{a^{2}+b^{2}}\right)}{\pi \sqrt{a^{2}+b^{2}}} .
$$

The mean length of $\mathrm{L}$ line per unit volume can now be written as the average

$$
d_{\mathrm{L}}=\left\langle\frac{\delta(|\boldsymbol{P} \times \boldsymbol{Q}|)}{\pi|\boldsymbol{P} \times \boldsymbol{Q}|}|\boldsymbol{A} \times \boldsymbol{B}|\right\rangle .
$$

Now the procedure is similar to that of the last section. We define the conditional probability density $\rho(\boldsymbol{A}, \boldsymbol{B} ; \boldsymbol{P}, \boldsymbol{Q})$ of the quantities $\boldsymbol{A}$ and $\boldsymbol{B}$ defined in (A 1) and (A 2), for $\boldsymbol{P}$ and $\boldsymbol{Q}$ fixed (and parallel). Thus

$$
\rho(\boldsymbol{A}, \boldsymbol{B} ; \boldsymbol{P}, \boldsymbol{Q})=\left\langle\delta\left(\boldsymbol{A}+P \nabla Q_{y}-Q \nabla P_{y}\right) \delta\left(\boldsymbol{B}-P \nabla Q_{x}+Q \nabla P_{x}\right)\right\rangle_{(\boldsymbol{P}, \boldsymbol{Q})},
$$

and so (6.3) can be written as

$$
d_{\mathrm{L}}=\int \mathrm{d} \boldsymbol{P} \int \mathrm{d} \boldsymbol{Q} \rho(\boldsymbol{P}) \rho(\boldsymbol{Q}) \frac{\delta(|\boldsymbol{P} \times \boldsymbol{Q}|)}{\pi|\boldsymbol{P} \times \boldsymbol{Q}|} \int \mathrm{d} \boldsymbol{A} \int \mathrm{d} \boldsymbol{B} \rho(\boldsymbol{A}, \boldsymbol{B} ; \boldsymbol{P}, \boldsymbol{Q})|\boldsymbol{A} \times \boldsymbol{B}| .
$$

The single-vector densities are given by (4.9). We calculate (6.4) by writing the $\delta$-functions as Fourier integrals and evaluating the resulting average (cf. (5.4)). Thus

$$
\rho(\boldsymbol{A}, \boldsymbol{B} ; \boldsymbol{P}, \boldsymbol{Q})=\frac{1}{(2 \pi)^{6}} \int \mathrm{d} \boldsymbol{s} \int \mathrm{d} \boldsymbol{t} \exp \{-\mathrm{i}(\boldsymbol{A} \cdot \boldsymbol{s}+\boldsymbol{B} \cdot \boldsymbol{t})\} \exp \left\{-\frac{1}{2} F\right\},
$$


where

$$
F=\left\langle\left[\boldsymbol{s} \cdot\left(-P \nabla Q_{y}+Q \nabla P_{y}\right)+\boldsymbol{t} \cdot\left(P \nabla Q_{x}-Q \nabla P_{x}\right)\right]^{2}\right\rangle_{(\boldsymbol{P}, \boldsymbol{Q})} .
$$

The averages over derivatives are given in (4.10), which, after exploiting the fact that $\boldsymbol{P}$ and $\boldsymbol{Q}$ are parallel, lead to

$$
F=k_{2}\left(P^{2}+Q^{2}\right)\left[\frac{1}{30}\left(s_{y}^{2}+t_{x}^{2}-s_{x} t_{y}-s_{y} t_{x}\right)+\frac{1}{15}\left(s_{x}^{2}+s_{z}^{2}+t_{y}^{2}+t_{z}^{2}\right)\right] .
$$

The $s$ and $\boldsymbol{t}$ integrals in (6.6) are now Gaussian, and give

$$
\rho(\boldsymbol{A}, \boldsymbol{B} ; \boldsymbol{P}, \boldsymbol{Q})=\frac{450 \sqrt{ } 5}{\left[\pi k_{2}\left(P^{2}+Q^{2}\right)\right]^{3}} \exp \left\{-\frac{H(\boldsymbol{A}, \boldsymbol{B})}{2 k_{2}\left(P^{2}+Q^{2}\right)}\right\},
$$

where

$$
H(\boldsymbol{A}, \boldsymbol{B})=16\left(A_{x}^{2}+B_{y}^{2}\right)+8 A_{x} B_{y}+40\left(A_{y}^{2}+B_{x}^{2}+A_{y} B_{x}\right)+15\left(A_{z}^{2}+B_{z}^{2}\right) .
$$

Rescaling $\boldsymbol{A}$ and $\boldsymbol{B}$ in (6.5) enables the $\boldsymbol{P}$ and $\boldsymbol{Q}$ integrals to be evaluated, using

$$
\int \mathrm{d} \boldsymbol{P} \int \mathrm{d} \boldsymbol{Q} \exp \left\{-3\left(P^{2}+Q^{2}\right)\right\} \frac{\left(P^{2}+Q^{2}\right)}{|\boldsymbol{P} \times \boldsymbol{Q}|} \delta(|\boldsymbol{P} \times \boldsymbol{Q}|)=\frac{2}{9} \pi^{3} .
$$

Thus the L line density becomes

$$
d_{\mathrm{L}}=\frac{2700 \sqrt{5} k_{2}}{\pi^{4}} \int \mathrm{d} \boldsymbol{A} \int \mathrm{d} \boldsymbol{B}|\boldsymbol{A} \times \boldsymbol{B}| \exp \left\{-\frac{1}{2} H(\boldsymbol{A}, \boldsymbol{B})\right\} .
$$

In Appendix D this six-fold integral is evaluated, giving the final result of this section,

$$
d_{\mathrm{L}}=0.21360 k_{2} \text {. }
$$

Comparison with (5.10) shows that the densities of $\mathrm{L}$ and $\mathrm{C}$ lines are nearly but not quite equal.

These predictions are in rough agreement with numerical calculations in figure 8 of Nye \& Hajnal (1987), showing a section of a monochromatic field of the form (4.1) containing six plane waves. The section, with area $100 / k^{2}$, is pierced by seven $\mathrm{C}$ lines and eight L lines, whereas (5.10) and (6.13) would predict about ten lines of each type.

\section{Concluding remarks}

We have studied the C and L lines identified (Nye \& Hajnal 1987) as the singularities of complex vector waves, that is, singularities of fields of polarization ellipses. But the following naive argument leads to the fallacious conclusion that the $\mathrm{L}$ singularities would be surfaces rather than lines. On the Poincaré sphere (Born \& Wolf 1959) that represents polarization, the two circular polarizations correspond to two points (the poles), while the linear polarizations correspond to a line (the equator), so $\mathrm{C}$ singularities would have codimension two, corresponding to lines in space, and L singularities would have codimension one, corresponding to surfaces in space.

The error lies in the use of the Poincaré sphere to describe waves that do not have a unique direction of propagation and so require all three spin- 1 components rather than the familiar two (cf. Berry 1987, §5). A correct version of the argument can 
be given in terms of the Majorana sphere (Hannay 1996, 1998), where a spin-1 state (e.g. light at a point) can be represented by two dots on a sphere. It can be shown (Hannay 1998) that circular polarization is represented by two coincident dots, and linear polarization by two antipodal dots. Both these situations are codimension two, leading to the correct prediction that $\mathrm{L}$, as well as $\mathrm{C}$, singularities are lines.

Although we have regarded $\boldsymbol{E}$ as the complex electric field vector, we do not claim that this is the final framework for describing the polarization singularities of light. Just as in Nye \& Hajnal (1987), we lack a description of singularities in which the magnetic and electric fields appear on an equal footing. It might be argued that the appropriate field to consider is the complex vector potential $\boldsymbol{A}$, but even after fixing the gauge (so that, for example, the scalar potential vanishes (Born \& Wolf 1959)) and observing that (for monochromatic waves at least) the $\mathrm{C}$ and $\mathrm{L}$ singularities of $\boldsymbol{A}$ correspond to those of the electric field, these are completely unrelated to those of the magnetic field.

Indeed, all our conclusions apply if $\boldsymbol{E}$ represents the magnetic field (or any other complex vector field). We do not regard this as a defect of the theory, but as a reflection of an important physical fact, recognized by Nye (1999) but not widely appreciated: for general (non-uniform) optical fields, polarization patterns of the electric and magnetic fields are different. Nevertheless, the statistics of singularities (for example, the $\mathrm{C}$ and $\mathrm{L}$ line densities that we have calculated) are the same for the electric and magnetic fields.

A pointer towards an intrinsically electromagnetic singularity theory could be the fact that many of our conclusions survive if the vectors $\boldsymbol{P}$ and $\boldsymbol{Q}$ are reinterpreted as the real electric and magnetic fields of light, so that $\boldsymbol{N}_{\mathrm{e}}$ in (2.3) is the instantaneous Poynting vector and the L singularities are loci of vanishing energy flow.

The possible existence of yet undiscovered singularities, where electric and magnetic fields are involved equally, should not be misinterpreted as implying that the electric C and L lines are unphysical. Indeed, electric (and magnetic) polarization singularities closely related to those we consider here have been observed in microwave experiments (Hajnal 1987a,b, 1990). (In this connection we should also point out that caustics and dislocations are also observable features of the physics of light, even though they dissolve under close examination.)

$\mathrm{C}$ and $\mathrm{L}$ line singularities are generic, that is, stable under perturbation. Intersection of two $\mathrm{C}$ lines is a codimension- 4 phenomenon, because from (2.4) this event requires the complex $\psi$, defined in (2.4), to vanish at two points. Such collisions can be expected to occur as collisions between $\mathrm{C}$ lines when an additional parameter is varied; in non-monochromatic waves this can happen naturally as time evolves. The local behaviour can be complicated, and the lines may change their topology. Similar arguments suggest that the intersection of two L lines is a codimension-4 phenomenon. By contrast, collisions between a $\mathrm{C}$ line and an $\mathrm{L}$ line are much harder to produce, because the polarization ellipse would have to be both a line and a circle; this is only possible if $\boldsymbol{E}=0$, which is a codimension-6 phenomenon and so is not expected to occur generically in space or spacetime (this event would be a singularity of the geometric phase 2-form, as explained after (3.9)). The two types of line singularity repel each other.

We emphasize that our considerations are essentially three dimensional: they apply to general waves in space, for which the propagation direction $\boldsymbol{k}(\boldsymbol{r})$ (equation (3.8)) varies with position. In paraxial waves, where there is a unique direction of propa- 
gation, different singularities can occur stably (Nye 1983, 1999). And, in the general case, although it is legitimate to regard places where $\mathrm{L}$ and $\mathrm{C}$ lines intersect a plane of observation as singular points, the labelling of these points by index or 'topological charge' must take account of the fact that the associated ellipses are not confined to the plane.

Finally, we draw attention to the fact that our statistical calculations of $\mathrm{C}$ and $\mathrm{L}$ line densities involve only the most elementary geometrical features of these singularities. It is possible to write expressions for the analogues of the other quantities calculated for dislocation lines in scalar waves (curvature, velocity and correlations between points where the lines intersect a plane (Berry \& Dennis 2000)), but in the vector case these take the form of multiple integrals so complicated that we have not been able to penetrate them analytically. In addition, we would like to be able to calculate statistics related to the propagation wavevector $\boldsymbol{k}$ (equation (3.8)), and also densities of two types of singular point peculiar to the vector case (Nye \& Hajnal 1987): where a $\mathrm{C}$ line touches its (circular) polarization ellipse (that is, $\boldsymbol{n}_{\mathrm{C}} \cdot \boldsymbol{n}_{\mathrm{e}}=0$ ) and the $\mathrm{C}$ index switches sign, and where an $\mathrm{L}$ line is perpendicular to its (linear) polarization ellipse (that is, $\boldsymbol{n}_{\mathrm{L}} \cdot \boldsymbol{e}_{\boldsymbol{P}}=0$ ) and the L index switches sign.

We thank Dr J. H. Hannay and Professor J. F. Nye for many helpful conversations. M.V.B. is supported by The Royal Society. M.R.D. is supported by a University of Bristol postgraduate scholarship.

\section{Appendix A. Direction (2.8) of an L line}

Let the origin $\boldsymbol{r}=0$ lie on an L line, with the vectors $\boldsymbol{P}$ and $\boldsymbol{Q}$ having the common direction $\boldsymbol{e}_{z}$. For small $\boldsymbol{r}$, we can expand

$$
\boldsymbol{P}(\boldsymbol{r})=P_{z} \boldsymbol{e}_{z}+\boldsymbol{a} \cdot \boldsymbol{r} \boldsymbol{e}_{x}+\boldsymbol{b} \cdot \boldsymbol{r} \boldsymbol{e}_{y}, \quad \boldsymbol{Q}(\boldsymbol{r})=Q_{z} \boldsymbol{e}_{z}+\boldsymbol{c} \cdot \boldsymbol{r} \boldsymbol{e}_{x}+\boldsymbol{d} \cdot \boldsymbol{r} \boldsymbol{e}_{y},
$$

where

$$
\boldsymbol{a}=\nabla P_{x}, \quad \boldsymbol{b}=\nabla P_{y}, \quad \boldsymbol{c}=\nabla Q_{x}, \quad \boldsymbol{d}=\nabla Q_{y} .
$$

Then the condition (2.7) that $\boldsymbol{r}$ lies on an L line becomes, using (2.3),

$$
\begin{aligned}
\boldsymbol{P}(\boldsymbol{r}) \times \boldsymbol{Q}(\boldsymbol{r}) & =\left(-P_{z} \boldsymbol{d}+Q_{z} \boldsymbol{b}\right) \cdot \boldsymbol{r} \boldsymbol{e}_{x}+\left(P_{z} \boldsymbol{c}-Q_{z} \boldsymbol{a}\right) \cdot \boldsymbol{r} \boldsymbol{e}_{y} \\
& \equiv \boldsymbol{A} \cdot \boldsymbol{r} \boldsymbol{e}_{x}+\boldsymbol{B} \cdot \boldsymbol{r} \boldsymbol{e}_{y}=\mathbf{0} .
\end{aligned}
$$

This implies $\boldsymbol{A} \cdot \boldsymbol{r}=\boldsymbol{B} \cdot \boldsymbol{r}=0$, so that the direction of the L line is parallel to

$$
\begin{aligned}
\boldsymbol{n}_{\mathrm{L}} \propto \boldsymbol{A} \times \boldsymbol{B} & =\left(-P_{z} \nabla Q_{y}+Q_{z} \nabla P_{y}\right) \times\left(P_{z} \nabla Q_{x}-Q_{z} \nabla P_{x}\right) \\
& =\nabla(\boldsymbol{P} \times \boldsymbol{Q})_{x} \times \nabla(\boldsymbol{P} \times \boldsymbol{Q})_{y} \\
& =\frac{1}{2} \nabla_{a} \times \nabla_{b}(\boldsymbol{P} \times \boldsymbol{Q})_{a} \times(\boldsymbol{P} \times \boldsymbol{Q})_{b} \cdot \boldsymbol{e}_{\boldsymbol{P}},
\end{aligned}
$$

where the penultimate equality depends on the fact that the $x$ and $y$ components of $\boldsymbol{P}$ and $\boldsymbol{Q}$ vanish at $\boldsymbol{r}=0$. The desired equation (2.8) follows immediately.

\section{Appendix B. Averages of derivatives of $P$ and $Q$}

This is the derivation of (4.10). From (4.1) we have, averaging over the phases $\mu_{k}$,

$$
\left\langle\left|\partial_{z} E_{z}\right|^{2}\right\rangle=2\left\langle\left(\partial_{z} P_{z}\right)^{2}\right\rangle=\sum_{k} k_{z}^{2} a_{\boldsymbol{k}}^{2}\left\langle\left|\boldsymbol{e}_{z} \cdot \boldsymbol{n}_{\boldsymbol{k}}\right|^{2}\right\rangle
$$


The average is over polarizations; to evaluate it, we define explicitly the coordinate system 1, 2, 3 used to define $\boldsymbol{n}$ in (4.2),

$$
e_{1} \equiv \frac{e_{k} \times e_{z}}{\left|e_{k} \times e_{z}\right|}, \quad e_{2} \equiv e_{k} \times e_{1}, \quad e_{3} \equiv e_{k}
$$

If $\boldsymbol{k}$ has polar angles $\theta, \phi$ (we do not write the suffixes $\boldsymbol{k}$ ), then

$$
\left.\begin{array}{l}
\boldsymbol{e}_{1}=(\sin \phi, \cos \phi, 0), \\
\boldsymbol{e}_{2}=(-\cos \theta \cos \phi, \cos \theta \sin \phi, \sin \theta), \\
\boldsymbol{e}_{3}=(\sin \theta \cos \phi, \sin \theta \sin \phi, \cos \theta) .
\end{array}\right\}
$$

On using (4.4) and (4.5), and integrating over the angles of $\boldsymbol{k}$ and polarizations, equation (B 1) becomes

$$
\begin{aligned}
\left\langle\left|\partial_{z} E_{z}\right|^{2}\right\rangle & =\frac{k_{2}}{(4 \pi)^{2}} \int_{0}^{2 \pi} \mathrm{d} \phi \int_{0}^{\pi} \mathrm{d} \theta \sin \theta \cos ^{2} \theta \int_{0}^{2 \pi} \mathrm{d} \beta \int_{0}^{\pi} \mathrm{d} \alpha \sin \alpha\left(\sin \theta \sin \frac{1}{2} \alpha\right)^{2} \\
& =\frac{1}{15} k_{2}
\end{aligned}
$$

giving the first set of equations in (4.10). Similarly,

$$
\begin{aligned}
&\left\langle\left|\partial_{z} E_{x}\right|^{2}\right\rangle=2\left\langle\left(\partial_{z} P_{x}\right)^{2}\right\rangle \\
&=\sum_{\boldsymbol{k}} k_{z}^{2} a_{\boldsymbol{k}}^{2}\left\langle\left|\boldsymbol{e}_{x} \cdot \boldsymbol{n}_{\boldsymbol{k}}\right|^{2}\right\rangle \\
&=\frac{k_{2}}{(4 \pi)^{2}} \int_{0}^{2 \pi} \mathrm{d} \phi \int_{0}^{\pi} \mathrm{d} \theta \sin \theta \cos ^{2} \theta \int_{0}^{2 \pi} \mathrm{d} \beta \int_{0}^{\pi} \mathrm{d} \alpha \sin \alpha \\
& \quad \times\left|\sin \phi \cos \frac{1}{2} \alpha \exp \left(-\frac{1}{2} \mathrm{i} \beta\right)-\cos \theta \cos \phi \sin \frac{1}{2} \alpha \exp \left(\frac{1}{2} \mathrm{i} \beta\right)\right|^{2} \\
&=\frac{2}{15} k_{2},
\end{aligned}
$$

giving the second set of equations in (4.10).

The third set of equations in (4.10) follows from $\nabla \cdot \boldsymbol{P}=0$, whence

$$
\left\langle(\nabla \cdot \boldsymbol{P})^{2}\right\rangle=3\left\langle\left(\partial_{x} P_{x}\right)^{2}\right\rangle+6\left\langle\partial_{x} P_{x} \partial_{y} P_{y}\right\rangle=0,
$$

so that

$$
\left\langle\partial_{x} P_{x} \partial_{y} P_{y}\right\rangle=-\frac{1}{2}\left\langle\left(\partial_{x} P_{x}\right)^{2}\right\rangle
$$

\section{Appendix C. Evaluation of integral (5.9) for C line density}

We require

$$
I_{\mathrm{C}} \equiv \int \mathrm{d} \boldsymbol{U} \int \mathrm{d} \boldsymbol{V}|\boldsymbol{U} \times \boldsymbol{V}| \exp \left\{-\frac{1}{2}\left[U^{2}+V^{2}-\frac{1}{4}\left(U_{z}^{2}+U_{z}^{2}\right)\right]\right\} .
$$

The length of a vector $\boldsymbol{X}$ can be represented as

$$
|\boldsymbol{X}|=-\frac{1}{2 \pi^{2}} \int \mathrm{d} \boldsymbol{t} \frac{1}{t^{2}} \nabla_{\boldsymbol{t}}^{2} \exp (\mathrm{i} \boldsymbol{X} \cdot \boldsymbol{t})
$$


Thus the $\boldsymbol{U}$ and $\boldsymbol{V}$ integrals are Gaussians, giving

$$
I_{\mathrm{C}}=-4 \pi \int \mathrm{d} t \frac{1}{t^{2}} \nabla_{t}^{2} \frac{1}{\sqrt{\operatorname{det} M}}
$$

where $M$ is the matrix

$$
M=\left(\begin{array}{cccccc}
1 & 0 & 0 & 0 & \mathrm{i} t_{z} & -\mathrm{i} t_{y} \\
0 & 1 & 0 & -\mathrm{i} t_{z} & 0 & \mathrm{i} t_{x} \\
0 & 0 & \frac{3}{4} & \mathrm{i} t_{y} & -\mathrm{i} t_{x} & 0 \\
0 & -\mathrm{i} t_{z} & \mathrm{i} t_{y} & 1 & 0 & 0 \\
\mathrm{i} t_{z} & 0 & -\mathrm{i} t_{x} & 0 & 1 & 0 \\
-\mathrm{i} t_{y} & \mathrm{i} t_{x} & 0 & 0 & 0 & \frac{3}{4}
\end{array}\right)
$$

A short calculation gives

$$
I_{\mathrm{C}}=-32 \pi \int \mathrm{d} \boldsymbol{t} \frac{\left[-33+20\left(t_{x}^{2}+t_{y}^{2}\right)+3 t_{z}^{2}\right]}{\left(t_{x}^{2}+t_{y}^{2}+t_{z}^{2}\right)\left[3+4\left(t_{x}^{2}+t_{y}^{2}\right)+3 t_{z}^{2}\right]^{3}} .
$$

This integral can be evaluated in cylindrical polar coordinates. The azimuthal integral is trivial, and the radial integral can be determined by elementary methods, leading to

$$
I_{\mathrm{C}}=-32 \pi^{2} \int \mathrm{d} t[g(t)+h(t)]
$$

where

$$
g(t)=\frac{\left(19+16 t^{2}+5 t^{4}\right)}{\left(t^{2}-3\right)^{3}\left(1+t^{2}\right)^{2}}, \quad h(t)=\frac{\left(33+17 t^{2}\right)}{\left(t^{2}-3\right)^{2}} \log \left\{\frac{3\left(1+t^{2}\right)}{4 t^{2}}\right\} .
$$

Although both $f(t)$ and $g(t)$ have singularities on the real axis at $t= \pm \sqrt{ } 3$, the sum $f(t)+g(t)$ is regular. Therefore, the $t$-contour can be deformed from the real axis to the curve $\Gamma$, descending in the upper left half-plane, touching the origin horizontally and ascending in the upper right half-plane. For the integral over $g(t)$, $\Gamma$ can be further deformed to enclose the double pole at $t=\mathrm{i}$, leading to

$$
\int_{\Gamma} \mathrm{d} \operatorname{tg}(t)=\frac{7}{8} \pi
$$

In $h(t)$, there are logarithmic branch points at $t=0$ and $t= \pm \mathrm{i}$; the correct phase is determined by replacing $t^{2}$ by $t^{2}+\varepsilon^{2}$, and $\Gamma$ deformed to enclose the branch cut between $t=\mathrm{i} \varepsilon$ and $t=\mathrm{i}$, leading to

$$
\int_{\Gamma} \mathrm{d} t h(t)=-2 \pi \int_{0}^{1} \mathrm{~d} y \frac{\left(33-17 y^{2}\right)}{\left(y^{2}+3\right)^{3}}=-\frac{29}{24} \pi-\frac{2 \pi^{2}}{9 \sqrt{ } 3} .
$$

Thus

$$
I_{\mathrm{C}}=32 \pi^{3}\left(\frac{1}{3}+\frac{2 \pi}{9 \sqrt{ } 3}\right),
$$

from which (5.10) follows immediately. 


\section{Appendix D. Evaluation of integral (6.12) for L line density}

We require

$$
I_{\mathrm{L}} \equiv \int \mathrm{d} \boldsymbol{A} \int \mathrm{d} \boldsymbol{B}|\boldsymbol{A} \times \boldsymbol{B}| \exp \left\{-\frac{1}{2} H(\boldsymbol{A}, \boldsymbol{B})\right\},
$$

with $H(\boldsymbol{A}, \boldsymbol{B})$ given by (6.10). Following the procedure for the similar (but simpler) integral (C 1), we use the identity (C 2) and evaluate the resulting Gaussian integrals in $\boldsymbol{A}$ and $\boldsymbol{B}$. This leads to

$$
I_{\mathrm{L}}=-\frac{8 \pi}{\sqrt{ } 5} \int \mathrm{d} \boldsymbol{t} \frac{u(\xi, \tau)}{t^{2} v(\xi, \tau)},
$$

where

$$
\left.\begin{array}{rl}
\xi \equiv & t_{x}^{2}+t_{y}^{2}, \\
\tau \equiv & t_{z}, \\
u(\xi, \tau) \equiv & 22016 \xi^{3}+192 \xi^{2}\left(-8400-40 \mathrm{i} \tau+169 \tau^{2}\right) \\
& +36 \xi\left(-205920000+768000 \mathrm{i} \tau-349600 \tau^{2}-800 \mathrm{i} \tau^{3}+277 \tau^{4}\right) \\
& +135(20 \mathrm{i}+\tau)^{2}\left(30240000+2275200 \mathrm{i} \tau-68640 \tau^{2}+392 \mathrm{i} \tau^{3}-11 t^{4}\right), \\
v(\xi, \tau) \equiv & {\left[128 \xi^{2}+24 \xi\left(3600-40 \mathrm{i} \tau+7 \tau^{2}\right)+45(20 \mathrm{i}+\tau)^{2}\left(-720-72 \mathrm{i} \tau+\tau^{2}\right)\right]^{5 / 2} .}
\end{array}\right\}
$$

The integral can be evaluated in cylindrical polar coordinates. The azimuthal integral is trivial and the radial integral is elementary (albeit complicated). This leaves the integral over $\tau$ as the sum over a part that can be evaluated by residues and a second part, namely,

$$
I_{\mathrm{L}}=\frac{\pi^{3}(87 \sqrt{10}-\sqrt{254419 / 5+77285 \sqrt{5} / 2})}{864000}-\frac{1}{\pi} \int_{-\infty}^{\infty} \mathrm{d} \tau \frac{\alpha(\tau)}{\beta(\tau)} \log \frac{\gamma(\tau)}{\delta(\tau)},
$$

where

$$
\left.\begin{array}{c}
\alpha(\tau)=8 \pi^{3}(5443200000 \mathrm{i}+225504000 \tau \\
\left.-13478400 \mathrm{i} \tau^{2}-599040 \tau^{3}+9372 \mathrm{i} \tau^{4}+205 \tau^{5}\right), \\
\beta(\tau)=25[(\tau+12 \mathrm{i}-24)(\tau+12 \mathrm{i}+24)]^{5 / 2}(\tau-60 \mathrm{i})^{4}, \\
\gamma(\tau)=4 \tau^{2}\left[-10800+120 \mathrm{i}+11 \tau^{2}\right. \\
-2 \sqrt{10} \operatorname{sgn} \tau \sqrt{(\tau+12 \mathrm{i}-24)(\tau+12 \mathrm{i}+24)}(\tau-60 \mathrm{i})], \\
\delta(\tau)=3(\tau+20 \mathrm{i})\left[216000 \mathrm{i}-10800 \tau+60 \mathrm{i} \tau^{2}+13 \tau^{3}\right. \\
\left.-5(\tau-60 \mathrm{i})^{3 / 2} \sqrt{(\tau+12 \mathrm{i}-24)(\tau+12 \mathrm{i}+24)(\tau-12 \mathrm{i})}\right] .
\end{array}\right\}
$$

We have not been able to evaluate the second contribution to (D 4) analytically, but numerical evaluation is easy and gives

$$
I_{\mathrm{L}}=-0.00342397+0.00687019=0.00344622 .
$$

The result (6.13) follows immediately. 


\section{References}

Allen, L., Padgett, M. J. \& Babiker, M. 1999 The orbital angular momentum of light. Prog. Opt. 39, 291-372.

Arnold, V. I. 1986 Catastrophe theory. Springer.

Berry, M. V. 1984 Quantal phase factors accompanying adiabatic changes. Proc. R. Soc. Lond. A 392, 45-57.

Berry, M. V. 1987 The adiabatic phase and Panacharatnam's phase for polarized light. J. Mod. Opt. 34, 1401-1407.

Berry, M. V. 1998 Paraxial beams of spinning light. In Singular optics (ed. M. S. Soskin), vol. 3487, pp. 6-13. Wahington, DC: SPIE.

Berry, M. V. 2000 Making waves in physics: three wave singularities from the miraculous 1830s. Nature 403, 21.

Berry, M. V. \& Dennis, M. R. 2000 Phase singularities in isotropic random waves. Proc. R. Soc. Lond. A456, 2059-2079 (errata 456, 3050).

Berry, M. V. \& Upstill, C. 1980 Catastrophe optics: morphologies of caustics and their diffraction patterns. Prog. Opt. 18, 257-346.

Born, M. \& Wolf, E. 1959 Principles of optics. Oxford: Pergamon.

Freund, I. 2000 Optical vortex trajectories. Opt. Commun. 181, 19-33.

Hajnal, J. V. $1987 a$ Singularities in the transverse fields of electromagnetic waves. II. Observations on the electric field. Proc. R. Soc. Lond. A 414, 447-468.

Hajnal, J. V. $1987 b$ Singularities in the transverse fields of electromagnetic waves. I. Theory. Proc. R. Soc. Lond. A 414, 433-446.

Hajnal, J. V. 1990 Observation of singularities in the electric and magnetic fields of freely propagating microwaves. Proc. R. Soc. Lond. A 430, 413-421.

Hannay, J. H. 1996 Chaotic analytic zero points: exact statistics for those of a random spin state. J. Phys. A 29, 101-105.

Hannay, J. H. 1998 The Majorana representation of polarization, and the Berry phase of light. J. Mod. Opt. 45, 1001-1008.

Mandel, L. \& Wolf, E. 1995 Optical coherence and quantum optics. Cambridge University Press.

Nye, J. F. 1983 Lines of circular polarization in electromagnetic wave fields. Proc. R. Soc. Lond. A 389, 279-290.

Nye, J. F. 1991 Phase gradient and crystal-like geometry in electromagnetic and elastic wavefields. In Sir Charles Frank, OBE: an eightieth birthday tribute (ed. R. G. Chambers, J. E. Enderby, A. Keller, A. R. Lang \& J. W. Steeds), pp. 220-231. Bristol: Adam Hilger.

Nye, J. F. 1999 Natural focusing and fine structure of light: caustics and wave dislocations. Bristol: Institute of Physics.

Nye, J. F. \& Berry, M. V. 1974 Dislocations in wave trains. Proc. R. Soc. Lond. A 336, 165-90.

Nye, J. F. \& Hajnal, J. V. 1987 The wave structure of monochromatic electromagnetic radiation. Proc. R. Soc. Lond. A 409, 21-36.

Shapere, A. \& Wilczek, F. (eds) 1989 Geometric phases in physics. World Scientific.

Simpson, N. B., Dholakia, A., Allen, L. \& Padgett, M. J. 1997 Mechanical equivalent of spin and orbital angular momentum of light: an optical spanner. Opt. Lett. 22, 52-54.

Soskin, M. S. (ed.) 1997 Singular optics, vol. 3487. Washington, DC: SPIE.

Vasnetsov, M. \& Staliunas, K. (eds) 1999 Optical vortices. Commack, NY: Nova Science. 